\title{
BISPECIFIC ANTIBODY PRETARGETING FOR IMPROVING CANCER IMAGING AND THERAPY
}

\author{
Final Report \\ DE-FG02-95ER62028
}

The main objective of this project was to evaluate pretargeting systems that use a bispecific antibody (bsMAb) to improve the detection and treatment of cancer. A bsMAb has specificity to a tumor antigen, which is used to bind the tumor, while the other specificity is to a peptide that can be radiolabeled. Pretargeting is the process by which the unlabeled bsMAb is given first, and after a sufficient time (1-2 days) is given for it to localize in the tumor and clear from the blood, a small molecular weight radiolabeled peptide is given. According to a dynamic imaging study using a $99 \mathrm{mTc}$-labeled peptide, the radiolabeled peptide localizes in the tumor in less than 1 hour, with $>80 \%$ of it clearing from the blood and body within this same time. Tumor/nontumor targeting ratios that are nearly 50 times better than that with a directly radiolabeled Fab' fragment have been observed (Sharkey et al., "Signal amplification in molecular imaging by a multivalent bispecific nanobody" submitted). The bsMAbs used in this project have been composed of 3 antibodies that will target antigens found in colorectal and pancreatic cancers (CEA, CSAp, and MUC1). For the "peptide binding moiety" of the bsMAb, we initially examined an antibody directed to DOTA, but subsequently focused on another antibody directed against a novel compound, HSG (histamine-succinyl-glycine).

\section{Major Accomplishments:}

The initial goal of this project was the development of a pretargeting system based on the LG-1 anti-DOTA antibody. A chemically conjugated Fab' x Fab' bispecific antibody (bsMAb) was prepared with an anti-CEA (carcinoembryonic antigen) antibody and targeting examined in nude mice bearing colorectal cancer xenografts (Losman et al., Proc. Am. Assoc. for Cancer Res., $44: 1290,2003$ ). Although reasonable targeting of an $111 \mathrm{In}$-labeled peptide was achieved within 3 hours, over time the radioactivity washed out of the tumor more quickly than other pretargeting systems using the same anti-CEA antibody, but different anti-hapten antibodies. Ultimately, BIAcore analysis showed that the affinity of LG1 Fab' was significantly reduced compared to the IgG. Further analysis showed LG-1 was binding specifically to the DOTA, but it also required a lysine linkage. This suggested that LG-1 not only recognized the DOTA, but that its specificity likely required some portion of the carrier that was used as the immunogen. An attempt was made to develop a new anti-DOTA antibody. For this purpose, two groups of mice were immunized, one with KLH (keyhole limpet hemocyanin) conjugated to peptide IMP 258 (Ac-Lys(DOTA)-Cys- $\mathrm{NH}_{2}$ ) through a thioether linkage, and the other with an IgG-DOTA conjugate that was used to prepare the LG- 1 anti-DOTA antibody. The former represented an attempt to develop an antibody response more specific for a DOTA-peptide that could be used in pretargeting, whereas the former simply looked to reproduce our previous attempt. Following fusion, cloning and recloning, at least 10 possible candidate clones were identified from the DOTA-peptide immunogen, but after recloning, either the clones were not stable or the antibody binding properties were not sufficient to warrant further development. Thus, we formally closed this line of investigation and more aggressively pursued another pretargeting systems using the anti-HSG (histamine-succinyl-glycine) hapten bindings

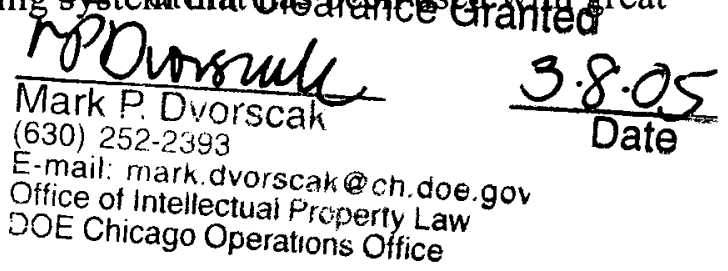




\section{DISCLAIMER}

This report was prepared as an account of work sponsored by an agency of the United States Government. Neither the United States Government nor any agency Thereof, nor any of their employees, makes any warranty, express or implied, or assumes any legal liability or responsibility for the accuracy, completeness, or usefulness of any information, apparatus, product, or process disclosed, or represents that its use would not infringe privately owned rights. Reference herein to any specific commercial product, process, or service by trade name, trademark, manufacturer, or otherwise does not necessarily constitute or imply its endorsement, recommendation, or favoring by the United States Government or any agency thereof. The views and opinions of authors expressed herein do not necessarily state or reflect those of the United States Government or any agency thereof. 


\section{DISCLAIMER}

Portions of this document may be illegible in electronic image products. Images are produced from the best available original document. 
success (Sharkey et al., Cancer Res., 63:354-63, 2003). Using this system in conjunction with an anti-CEA antibody for tumor targeting, extended biodistribution studies have shown pretargeting is able to deliver about 1.5 - to 2.0 - times the radiation dose to a tumor as a directly radiolabeled anti-CEA antibody at equitoxic doses, with a dose rate that is 3 times that of a 90Y-labeled IgG (Sharkey et al., Clin. Cancer Res., 9:3897s-3913s, 2003). In addition, the dose rate to the tumor is nearly tripled. This pretargeting system, now using a novel recombinant trivalent bsMAb (Rossi et al., "Pretargeting of CEA-expressing cancers with a trivalent bispecific fusion protein produced in myeloma cells." Clin Cancer Res., in press). Over the last year, a number of therapy studies have been performed in the GW-39 colorectal cancer model that examined whether pretargeting using the anti-CEA bsMAb and a $90 \mathrm{Y}$-labeled peptide was superior to a $90 \mathrm{Y}$ labeled anti-CEA IgG. These studies have included determinations of maximally tolerated doses in the nude mice for both the $90 \mathrm{Y}-\mathrm{IgG}$ and the $90 \mathrm{Y}$-peptide under optimal pretargeting conditions. Therapy studies were then set up to examine a range of $90 \mathrm{Y}$-radioactivity doses proportional to the maximum tolerated dose in animals bearing subcutaneous tumors of varying size. Both of these treatments are fairly effective in nude mouse models, and therefore observations are continuing because an extended period of time is required to observe potential differences. One study was terminated after 14 weeks, because the $90 \mathrm{Y}-\mathrm{IgG}$ dose given in this study was beyond the MTD (i.e., lead to several treatment-related deaths), whereas the dose of $90 Y-l a b e l e d$ peptide was well tolerated. Thus, continued comparison of efficacy in this study would not have fairly compared each treatment at equitoxic doses of radioactivity (i.e., the 90YIgG group was overdosed). Nevertheless, in examining survival as defined by the time it took for tumors to reach $2.5 \mathrm{~cm} 3$ (starting tumor size was $0.5 \mathrm{~cm} 3$ ), at 14 weeks, $7 / 10$ animals in the pretargeting group had tumors $<2.5 \mathrm{~cm}^{3}$, whereas the median "survival" for the ${ }^{90} \mathrm{Y}$-IgG group was reached. Histological examination of the 7 tumor masses taken from the pretargeting group revealed 3 were completed devoid of viable tissue. Thus, this pretargeting treatment, which was safely tolerated in all animals, resulted in a $30 \%$ cure rate of substantially sized $(0.7$ grams) tumors. Two of the other 4 tumors had only a single microscopic focus of viable tumor, which would have taken many more weeks to progress to $2.5 \mathrm{~cm}^{3}$. The other 2 tumors were composed of approximately 30 to $70 \%$ viable tumor cells, but they were $\sim 1.0 \mathrm{~cm}^{3}$ (they had reached this size just 2-weeks after treatment when all growth was arrested), and therefore would have required several more weeks before reaching $2.5 \mathrm{~cm}^{3}$. Thus, although $50 \%$ of the surviving animals in the ${ }^{90} \mathrm{Y}$-IgG group had reached a tumor size of $2.5 \mathrm{~cm}^{3}$ at the termination of the study (median survival 14 weeks), the median survival for the ${ }^{90} \mathrm{Y}$-peptide pretargeting group had not been reached at this time, and would have likely extended considerably longer had the study not been terminated.

Several other studies have now been completed with monitoring periods as long a 36-weeks. As in these earlier studies, the data continue to support the improved therapeutic outcome with the pretargeting approach compared to the direct targeting.

\section{Pretargeting Colorectal Cancer using Mu-9 anti-CSAp bsMAb}

Mu-9 is a MAb directed against an antigen found primarily in colorectal cancers. Preclinical studies showed improved targeting and retention of radiolabeled $\mathrm{Mu}-9$ compared to our antiCEA antibodies, suggesting that $\mathrm{Mu}-9$ might have an advantage in the therapy of colorectal cancer. Thus, our objective was to determine whether a bsMAb composed of Mu9 would have 
an advantage over bsMAb directed to CEA for pretargeting. A humanized version of $\mathrm{Mu}-9$ has been prepared and this was used to make hMu9 x m679 Fab' x Fab' bsMAb. Biodistribution studies in nude mice bearing colorectal cancer xenografts have been performed with the directly radioiodinated IgG, F(ab') $)_{2}$, Fab' of Mu-9 and the bsMAb. The F(ab') ${ }_{2}$ and the Fab' fragments cleared rapidly from blood, and there was evidence that the $F\left(a b^{\prime}\right)_{2}$ was not stable in the blood. However, studies with the chemically stabilized Mu-9 Fab' x Fab' bsMAb showed the expected stability that has been observed with other bsMAb prepared using this method. When used in pretargeting, tumor uptake an ${ }^{111}$ In-labeled peptide was $23.5 \pm 4.4 \%$ injected dose (ID)/g within $3 \mathrm{~h}$ and was maintained at this level for 2 days, and even 5 days later, $13.6 \pm 3.6 \% \mathrm{ID} / \mathrm{g}$ remained in the tumor. Tumor/nontumor ratios at $3 \mathrm{~h}$ were 9:1, 5:1, and $>25: 1$ for the blood, kidney, and other major organs. Analysis of area under the curve (AUC) over 5 days showed pretargeting with the Mu-9 bsMAb was nearly 2-fold higher than that seen with pretargeting using the directly radiolabeled $\mathrm{Mu}-9 \mathrm{IgG}$ over a similar period, and with a 3-fold higher radiation dose rate. Tumor/nontumor ratios based on the AUC were 45:1, 8:1, and 32:1 for the blood, kidney, and liver; respectively However, it is equally important to note that this represents a significant improved compared to the excellent pretargeting results achieved with the anti-CEA bsMAb pretargeting system.

\section{Pretargeting Pancreatic Cancer cPAM4 anti-MUC-1 x m734 anti-(In)DTPA}

The primary rationale for exploring pretargeting in pancreatic cancer is the recent work by investigators at our institution with a 90Y-labeled anti-MUC-1 antibody, PAM4. In preclinical studies, treatment of CAPAN1 human pancreatic cancer xenografts in nude mice with $260 \mathrm{uCi}$ of

${ }^{90} \mathrm{Y}$-labeled cPAM4 IgG showed effective anti-tumor effects. However, more importantly, when only $25 \mathrm{uCi}$ of $90 \mathrm{Y}-\mathrm{cPAM} 4 \mathrm{IgG}$ (that delivered a dose of only $800 \mathrm{cGy}$ to the tumor) was combined with a full course of gemcitabine, the only approved treatment for pancreatic cancer that also serves as a radiosensitizer, significantly improved anti-tumor effects were achieved (Gold et al., Clin. Cancer Res., 3929s-3937s, 2003). 90Y-hPAM4 IgG is now being studied clinically, but we already are anticipating that a pretargeting approach with PAM4 will have the added advantage of reducing hematologic toxicity, but with the ability to deliver the same or more radiation dose to the tumor as the radiolabeled IgG. This is important since gemcitabine is myelosuppressive.

A bsMAb using cPAM4 Fab' and the anti-hapten antibody m734, an anti-indium-loaded DTPA antibody, was prepared and pretargeting tested in nude mice bearing human $\mathrm{CaPan} 1$ pancreatic cancer xenografts (Cardillo et al., Clin Cancer Res., 10:3552-3461, 2004). Biodistribution studies of the bsMAb alone and pretargeting studies using a ${ }^{99 m} \mathrm{Tc}$ - and an ${ }^{111} \mathrm{In}$-labeled peptide were performed. High tumor uptake ( $\sim 20 \% \mathrm{ID} / \mathrm{g}$ at $3 \mathrm{~h}$ post peptide injection) and retention was observed with both of these peptides. Peptide tumor uptake was negligible with the control group pretargeted with the non-specific bsMAb and also in a group that received the peptide alone. Peptide tumor/non-tumor ratios were high as early as $3 \mathrm{~h}$ post peptide injection. These studies show promise for imaging and therapy of pancreatic cancer with pretargeting. We are in the process of preparing bsMAb with the PAM4 and anti-HSG antibody to evaluate therapeutic issues for pancreatic cancer. 
Importantly, each of the targeting systems listed above, CEA, CSAp and MUC1 can be used in pancreatic cancer. Thus, we are also planning to pursue an evaluation of using this trio of bsMAb for targeting pancreatic cancer in animal models. However, while we have shown each of these antigens to be present in fresh human pancreatic cancer tissue, we will first need to select appropriate cell lines that express all 3 antigens to pursue this line of investigation.

\section{Treatment of Non-Hodgkin's Lymphoma using hA20 anti-CD20 x m679 anti-HSG bsMAb}

Based on the encouraging therapeutic advantage reported by investigators at the University of Washington with a streptavidin-anti-CD20 pretargeting system in NHL, we decided to explore the potential of the bsMAb pretargeting approach for NHL. Our rationale for pursuing this approach is that streptavidin is immunogenic, even in NHL patients, and thus, by using a bsMAb that is humanized, we would avoid, or at least significantly reduce this problem. In addition, although 2 FDA-approved anti-CD20 antibody therapeutics are already available for NHL, the data from the University of Washington suggest that pretargeting should have a therapeutic advantage with less toxicity. Our goal is to assess whether a bsMAb pretargeting approach could achieve similar success.

A bsMAb using hA20, a humanized antibody to CD20 was prepared with the anti-HSG antibody. The biodistribution of the bsMAb alone and in a pretargeting system with an $111 \mathrm{In}$-labeled peptide have been examined in SCID mice bearing Raji human Burkett's lymphoma cell line. In pretargeting, peptide uptake was similar to values reported by the University of Washington investigators with the Ramos xenografts bearing mice, but with even better tumor/blood ratios. Three pilot therapy studies in nude mice bearing $\geq 1.0 \mathrm{~cm}$ diameter Ramos human B-cell lymphoma xenografts have shown the pretargeting approach using 90Y-labeled peptide is superior to the directly $90 \mathrm{Y}$-labeled anti-CD20 IgG in the Ramos B-cell lymphoma cell line. [Sharkey et al., "Improving the treatment of non-Hodgkin's lymphoma using radionuclides pretargeted with a new anti-CD20 bispecific antibody." (submitted).] Thus, these preliminary studies confirm the superiority of pretargeting in B-cell lymphoma using the anti-CD20 $\mathrm{x}$ antiHSG pretargeting system.

Publications: Over the course of this project, a number of abstracts were submitted for presentation to national meetings (SNM, AACR, etc.). The listing below shows several articles have been published or recently submitted. We are in the process of preparing 2 additional manuscripts that will describe (a) a comparison of the targeting with various forms of molecularly engineered, recombinant bsMAb, and (b) summary of the therapy studies in the human colorectal cancer model comparing pretargeting and directly targeted antibodies.

\section{Articles:}

Cardillo TM, Karacay H, Goldenberg DM, Yeldell D, Chang CH, Modrak DE, Sharkey RM, Gold DV. Improved targeting of pancreatic cancer: experimental studies of a new bispecific antibody, pretargeting enhancement system for immunoscintigraphy. Clin Cancer Res. 2004 May 15;10(10):3552-61. 
Sharkey RM, Karacay H, Cardillo TM, Chang C-H, McBride WJ, Rossi EA, Horak ID, and Goldenberg DM. Improving the Delivery of Radionuclides for Imaging and Therapy of Cancer Using Pretargeting Methods. Clin Cancer Res. (in press).

Rossi EA, Chang C-H, Losman MJ, Sharkey RM, Karacay H, McBride WJ, Cardillo TM, Hansen HJ, Qu Z, Horak ID, and Goldenberg DM. Pretargeting of CEA-expressing Cancers with a Trivalent Bispecific Fusion Protein Produced in Myeloma Cells. Clin Cancer Res. (in press).

Sharkey RM, Karacay H, Chang C-H, McBride WJ, Horak ID, and Goldenberg DM. Improving the Treatment of Non-Hodgkin's Lymphoma Using Radionuclides Pretargeted with a New Anti-CD20 Bispecific Antibody (submitted).

\section{Abstracts:}

Cardillo TM, Karacay H, Schutsky K, McBride W, Goldenberg DM, Sharkey RM. Enhanced targeting of an ${ }^{111}$ In-labeled peptide in experimental pancreatic cancer with a pre-targeted $\mathrm{F}(\mathrm{ab})_{2}$ anti-MUC1 bispecific antibody. Cancer Biother Radiopharm 2002; 17(4): 478.

E.A. Rossi, CH Chang, RM Sharkey, W McBride, H Karacay, L Zeng, I Horak, HJ Hansen, DM Goldenberg. New bispecific, multivalent, scFv molecular constructs recombinant-engineered from anti-CEA antibody variable domains for Pretargeting cancer therapy. Presented at Keystone Symposia on Antibody-Based Therapeutics for Cancer. (Abstract \# 216), 2003.

Losman M, Govindan SV, Newsome GA, Karacay H, McBride WJ, Chang K, Griffiths GL, Hansen HJ, Goldenberg DM, Sharkey RM. Generation and characteristics of a mouse monoclonal anti-DOTA antibody, LG-1 for a bispecific antibody pretargeting system. Proc Am Assoc Cancer Res., 44; 1290, 2003.

Karacay H, Cardillo T, McBride WJ, Gold DV, Sharkey RM, Chang CH, Hansen, HJ, Goldenberg, DM. Pretargeting with bispecific antibody as a method for improved imaging of pancreatic cancer. J. Nucl. Med. 2003; 44(5 Suppl),176P.

Karacay H, Sharkey RM, Newsome G, Yeldell D, McBride WJ, Chang C-H, Hansen HJ, and Goldenberg DM. Bispecific antibody (bsMAb) pretargeting for the radioimmunotherapy (RAIT) of colorectal cancer using a bsMAb against colon-specific antigen-p (CSAp). Presented EORTC meeting, Boston, MA Nov 2003.

D.V. Gold, T.M. Cardillo, H. Karacay, R.M. Sharkey, K. Chang and D.M. Goldenberg. A pretargeted, bispecific antibody system for nuclear imaging of pancreatic cancer. Proc. Am. Assoc. Cancer Res. 44:1273 (Abstract \#6369), 2003.

R.M. Sharkey, H. Karacay, C.H. Change, L. Zeng, W.J. McBride I.D. Horak, H.J. Hansen, D.M. Goldenberg. Preclinical assessment of pretargeting therapy of non- 
Hodgkin's lymphoma (NHL) using a bispecific humanized anti-CD20 antibody and a ${ }^{90}$ Y-labeled peptide. J Nucl. Med. (Suppl.) 45, No. 5: 322P (Abstr. \#1019), 2004.

R.M. Sharkey, H. Karacay, E.A. Rossi, C.H. Chang, W. McBride, I.D. Horak, H.J. Hansen, D.M. Goldenberg. Recombinant bispecific antibodies (bsMAb) to carcinoembryonic antigen (CEA): Promising new agents for pretargeted radioimmunotherapy (RAIT) of solid tumors. Meeting Proceedings American Society of Clinical Oncology 23: 221 (Abstr. \#3105), 2004.

E.A. Rossi, S.K. Saha, R.M. Sharkey, H. Karacay, I.D. Horak, D.M. Goldenberg, C.H. Chang. A trivalent bispecific fusion protein engineered from antibody variable domains for improved pretargeting of CEA-expressing cancers. Proceedings of the AACR 45: 232 (Abstr. \#1019), 2004 